\title{
Análise dos fatores de risco na correção cirúrgica do defeito septal atrioventricular de forma total
}

\author{
Eduardo Keller SAADI*, Darryl SHORE**, Christopher LINCOLN ${ }^{\star *}$
}

RBCCV 44205-209

SAADI, E. K.; SHORE, D.; LINCOLN, C. - Análise dos fatores de risco na correção cirúrgica do defeito septal atrioventricular de forma total. Rev. Bras. Cir. Cardiovasc., 8(2):136-151, 1993.

RESUMO: Pacientes com defeito septal atrioventricular de forma total (DSAVT) freqũentemente apresentam insuficiência cardíaca intratável e hipertensão arterial pulmonar nos primeiros meses de vida, e apenas uma minoria sobrevive sem tratamento cirúrgico precoce. Por essa razăo, indica-se a correção definitiva para alterar favoravelmente a história natural da doença. Entretanto, vários fatores são responsáveis pela alta mortalidade cirúrgica. O presente trabalho estuda a experiência na correção cirúrgica do DSAVT com o objetivo de identificar alguns fatores de risco estatisticamente significativos para a ocorrência de morte operatória. Analisaram-se, retrospectivamente, 52 pacientes submetidos, entre janeiro de 1974 e dezembro de 1990, a cirurgia definitiva para correção de DSAVT no Royal Brompton and National Heart and Lung Institute, sendo estudadas as seguintes variáveis: idade, peso, sexo, ano da operaçăo, presença de síndrome de Down, grau de regurgitação da valva AV, bandagem prévia do tronco pulmonar, presença de anomalias associadas, pressão sistólica pulmonar, duplo orifício mitral, classificação do defeito segundo Rastelli, emprego de parada circulatória e técnica de correçăo ( $1 \times 2$ retalhos). Todos os fatores foram avaliados isoladamente, mediante a análise univariada. Para determinar quais os fatores que, independentemente da ação de outros, contribuíram significativamente para maior mortalidade cirúrgica, foi utilizada a análise multivariada com regressão logística. A análise multivariada demonstrou que o baixo peso na época da operação e a técnica de correção com um retalho aumentam significativamente a mortalidade cirúrgica.

DESCRITORES: defeito septal atrioventricular, cirurgia, fatores de risco.

\section{INTRODUÇÃO}

O defeito septal atrioventricular de forma total (DSAVT) é uma cardiopatia congênita complexa caracterizada por:

- Deficiência no septo atrial imediatamente acima das valvas atrioventriculares.

- Deficiência na via de entrada do septo ventricular, com a presença de comunicação interventricular.

- Presença de uma valva atrioventricular úni- ca, geralmente composta por 5 ou 6 cúspides $5,7,12,56$.

Os corações com esta anomalia apresentam, ainda, outras características morfológicas, como encurtamento da via de saída do ventrículo esquerdo, configuração anormal da via de saída do ventrículo esquerdo e perda da posição em cunha, da aorta, entre as válvas atrioventriculares $5-7,12,16,34$. $35,39,41,67,89-91,93,104,105,118,128,129,131$; há aumento de ambos os ventrículos.

Vários são os estudos, na literatura, referentes

Trabalho realizado no Royal Brompton and National Heart and Lung Hospital, Londres, Inglaterra.

Apresentado ao $20^{2}$ Congresso Nacional de Cirurgia Cardiaca. Maceió, AL, 2 e 3 de abril, 1993.

- Do Hospital de Clínicas de Porto Alegre, RS, Brasil. Senior Fellow. Royal Brompton and National Heart and Lung Hospital.

* Do Royal Brompton and National Heart and Lung Hospital.

Endereço para separatas: Eduardo Keller Saadi. Rua Honório Silveira Dias, 1500, apto. 503. 90540-070 Porto Alegre, RS, Brasil. 
SAADI, E. K.; SHORE, D.; LINCOLN, C. - Análise dos fatores de risco na correção cirúrgica do defeito septal atrioventricular de forma total. Rev. Bras. Cir. Cardiovasc., 8(2):136-151, 1993.

à morfologia e à classificação desta lesão, que, no passado, recebeu as designações de "defeitos do coxim endocárdico", "persistência do canal atrioventricular" e canal atrioventricular communis 16,73 , 96-100, 113, 116, 129, 131. Como padronizado por BECKER \& ANDERSON ${ }^{12}$, recentemente, é mais correto, do ponto de vista anatômico, caracterizar tais lesões como deficiência da septação atrioventricular.

A expressão "defeito septal atrioventricular" (DSAV) enfatiza a morfologia anormal da junção atrioventricular, que é a chave para a compreensão e a correção da lesão ${ }^{89}$.

Os termos "parcial" e "total", atribuídos a este defeito, foram introduzidos por WAKAI \& EDWARDS 124 , em 1956. Desde então, já havia consciência de que nem todos os casos poderiam ser incluídos nas duas categorias. BHARATI \& LEV ${ }^{16}$, anos mais tarde, insatisfeitos com a classificação do defeito apenas em "parcial" e "total", acrescentaram a forma "intermediária" ou "de transição".

Na mesma época, LEV ${ }^{65}$ descreveu a posição do nó atrioventricular e do feixe de His nesta doença. A diferença básica deve-se à ausência de estruturas septais atrioventriculares, sendo nesta área que, em corações normais, o nó atrioventricular dá origem ao feixe de condução. Devido à deficiência de tecido septal, a borda inferior do septo atrial faz contato com o septo ventricular apenas na crux cordis. O defeito no septo atrioventricular desloca o seio coronário inferiormente. $O$ nó atrioventricular também se desloca inferiormente, localizando-se na parede posterior do átrio direito por sobre a crux cordis, entre o orifício do seio coronário e a crista do septo ventricular, no chamado triângulo nodal 118 .

Durante a década de 60 , a possibilidade de tratamento cirúrgico desta lesão serviu de estímulo para estudos anatômicos mais detalhados 96 .

CARPENTIER ${ }^{22}$, em 1978, acrescentou a importância, na classificação dos defeitos, de se utilizarem termos morfológicos e não embriológicos, com o objetivo de descrever de maneira acurada o defeito e compreender a anatomia cirúrgica. A designação de "defeito septal atrioventricular" preencheria ambos os critérios.

Poucos são os estudos que se preocuparam com o delineamento da história natural do DSAVT $14,32,79$

BERGER et alii ${ }^{14}$ analisaram, através de autopsia, 39 pacientes com DSAVT e sem tratamento cirúrgico. Através de análise atuarial da idade no momento da morte, concluíram que apenas $54 \%$ das crianças que nasceram com este defeito estavam vivas aos 6 meses de idade, $35 \%$ aos 12 meses, $15 \%$ aos 24 meses e $4 \%$ aos 5 anos de idade.
A principal causa da morte nos primeiros 2 anos de vida foi insuficiência cardiaca congestiva, com ou sem infecção respiratória de repetição, como resultado de grande shunt esquerda-direita, insuficiência da valva atrioventricular e hipertensão pulmonar $53,83,112,114$.

Alguns autores observaram desenvolvimento mais rápido da doença vascular pulmonar em crianças com síndrome de Down e cardiopatia congênita quando comparadas com crianças sem síndrome de Down 63 .

CLAPP et alii ${ }^{26}$, comparando 45 pacientes com DSAVT e síndrome de Down, demonstraram que o grupo com síndrome de Down apresentou maior elevação da resistência vascular pulmonar durante o primeiro ano de vida e progressão mais rápida da doença vascular pulmonar obstrutiva fixa do que o grupo com cromossomas normais.

Alguns autores questionam a indicação cirúrgica em crianças com síndrome de Down 20 .

EMANUEL et alii ${ }^{36}$ observaram que os pacientes com DSAVT têm maior tendência de terem filhos com cardiopatia congênita.

Em análise de 52 crianças cujos pais apresentavam DSAV, foi detectada, em 5 , a presença de cardiopatia congênita. A mãe era cardiopata em todos os casos. Nesta série relativamente pequena, a incidência de cardiopatia congênita nos filhos de pais com defeito septal atrioventricular foi de $9,6 \%$ e de $14,3 \%$ quando somente a mãe era considerada. Tal incidência é bem mais alta do que a relatada em outras formas de cardiopatia congênita.

O aconselhamento genético em cardiopatias congênitas tem evoluído durante as últimas décadas ${ }^{84}$. Cerca de $8 \%$ dos casos se devem predominantemente a fatores genéticos e $2 \%$ sobretudo a fatores ambientais. Em $90 \%$ dos casos há considerável interação genético-ambiental.

Desde a introdução, por RASTELLI et alii 96-99, no final da década de 60 , da classificação anatômica e de um método cirúrgico para a correção dessa lesão, houve um grande avanço em seu tratamento cirúrgico. $\mathrm{Na}$ época em que começaram a ser realizados tais procedimentos, a mortalidade cirúrgica era superior a $60 \%$. Mais recentemente, houve grande melhora nos resultados cirúrgicos do reparo definitivo do DSAVT $13-15,18,51,72,76,77,85,113,114,116$, 120, 129.

O primeiro reparo bem sucedido de um defeito septal atrioventricular de forma total foi realizado por LILLEHEI et alii ${ }^{66}$, em 1954, usando circulação cruzada com a mãe da criança, sendo o defeito corrigido através de sutura direita da borda da comunicação interatrial com a crista do septo ventricular. 
Em 1958, com a precisa descrição da anatomia do tecido de condução, por LEV ${ }^{65}$, houve redução importante da incidência de bloqueio atrioventricular (AV) total. A partir de então, vários autores estudaram detalhadamente o tecido de condução neste defeito $38,47,60,61,78$. Até essa época, a mortalidade cirúrgica era alta, freqüentemente associada a bloqueio AV total, insuficiência severa da valva atrioventricular ou estenose subaórtica 56 .

Inicialmente, a maioria dos cirurgiões empregava a técnica de dois retalhos, tendo MALONEY Jr. et alii ${ }^{71}$, em 1962, descrito a técnica de apenas um retalho para fechar a comunicação interatrial, a comunicação intraventricular e ressuspender as cúspides valvares. Esta técnica levou a importante redução na mortalidade hospitalar 51,97 . Mais recentemente, contribuições importantes, como a de CARPENTIER ${ }^{22}$, principalmente por seus novos conceitos com relação às valvas atrioventriculares, e uma melhor compreensão deste complexo defeito e ao retorno da técnica de correção com dois retalhos, contribuíram para a melhora marcante do resultado cirúrgico.

KATZ et alii 52, em estudo experimental, demonstraram que são recomendáveis as suturas com reforço, por apresentarem menos risco de deiscência. Quando forem usados fios de sutura sem reforço, os pontos devem englobar grande quantidade de tecido.

KAWASHIMA et alii 54 acompanharam 8 pacientes submetidos a fechamento de comunicação interventricular (CIV) com retalho de pericárdio autólogo, durante o período de até 5 anos e 9 meses, tendo ocorrido aneurisma do retalho em 3 pacientes.

KIRKLIN et alii 58 fazem referência à mortalidade de apenas $2 \%$ de 41 pacientes operados, em 1984 e 1985, na Universidade de Alabama.

Nos últimos anos, estes excelentes resultados foram estendidos a formas complexas e defeitos associados, como tetralogia de Fallot ou dupla via de saída do ventrículo direito, entre outros $3,4,8,29$. $42,81,86-88,95,111,121-123,132$.

\section{O tratamento cirúrgico no DSAVT visa a:}

1) fechamento da comunicação interatrial;

2) fechamento da comunicação interventricular;

3) evitar lesão ao tecido de condução;

4) criação de duas valvas atrioventriculares não estenóticas e suficientes 7,56 .

A importância da criação de valva mitral não estenótica e sem insuficiência severa está bem demonstrada no prognóstico dos pacientes $43,56,114$.

Crianças e recém-nascidos com DSAVT freqüentemente têm sérias enfermidades, com insufi- ciência cardíaca intratável, infecções respiratórias de repetição e baixo peso, sendo difícil obter-se tratamento clínico efetivo ${ }^{9}$. Além disso, estão sujeitos ao desenvolvimento precoce de doença vascular pulmonar obstrutiva 27, 44, 45,83. Por causa desses indicadores de mau prognóstico, combinados com os recentes avanços nos campos de anestesia, proteção miocárdica, circulação extracorpórea e cuidados pós-operatórios, existe, atualmente, a tendência em favor da correção primária desta lesão na infância $1,2,10,13,15,25,28,55,57-59,70,74,114,127$.

A maioria dos artigos, na literatura, descreve os resultados cirúrgicos de maneira confusa, sem distinguir, na estatística, casos de defeito septal atrioventricular de forma parcial do tipo ostium primum de outros, como defeito septal atrioventricular de forma total $70,101,114$. Alguns autores incluem apenas casos de experiência bastante recente ou que foram tratados por método cirúrgico específico 77. 127. Todos esses fatores, associados à pouca freqũência e à complexidade da doença em questão, dificultam a avaliação de variáveis que influenciam no resultado cirúrgico.

A presente investigação tem como objetico analisar os potenciais fatores de risco de morte operatória em pacientes submetidos a correção definitiva do defeito septal atrioventricular de forma total.

\section{CASUÍSTICA E MÉTODOS}

$O$ estudo envolve todos os pacientes submetidos a correção definitiva do defeito septal atrioventricular de forma total no "Royal Brompton and National Heart and Lung Institute", em Londres, Inglaterra, que foram operados por dois cirurgiōes cardiovasculares pediátricos. Os critérios diagnósticos, tanto clínicos como de exames complementares, como ecocardiografia e cineangiografia, entre outros, estão bem descritos em diversos artigos 11,17 , $31,49,62,82,92,106,107,109,110,115$. A ecocardiografia fetal possibilita, nos dias de hoje, o diagnóstico prénatal desta cardiopatia 69 .

Foram classificados como defeitos de forma total aqueles em que esteve presente uma comunicação interventricular e houve necessidade de fechamento durante a operação, na presença de valva atrioventricular única. Utilizaram-se os critérios de classificação descritos por BECKER \& ANDERSON 12. Foram excluídos do estudo apenas os pacientes que apresentavam hipoplasia do ventrículo esquerdo nos quais a operação foi contra-indicada ou realizado outro procedimento tipo "Fontan".

Procedeu-se a detalhada revisão retrospectiva de todos os documentos hospitalares dos 52 pa- 
SAADI, E. K.; SHORE, D.; LINCOLN, C. - Análise dos fatores de risco na correção cirúrgica do defeito septal atrioventricular de forma total. Rev. Bras. Cir. Cardiovasc., 8(2):136-151, 1993.

cientes operados de janeiro de 1974 a dezembro de 1990.

\section{Analisaram-se as seguintes variáveis:}

Idade, sexo, anos da operação, peso, presença da síndrome de Down, grau de regurgitação da valva atrioventricular, bandagem prévia do tronco pulmonar, presença de duplo orifício "mitral", classificação do defeito segundo Rastelli, emprego de parada circulatória e técnica de correção do defeito.

Todas as variáveis foram testadas contra a variável dependente, que foi morte operatória. Considerou-se morte operatória a ocorrência de óbito durante a mesma internação hospitalar em que ocorreu o procedimento cirúrgico, ou nos 30 dias que se seguiram à operação.

Todos os pacientes foram operados com o auxílio de circulação extracorpórea, com graus variáveis de hipotermia. Todas as crianças com menos de $8 \mathrm{~kg}$ de peso foram submetidas a hipotermia de superfície até $28^{\circ} \mathrm{C}$ e, após, em circulação extracorpórea, através de canulação da aorta ascendente e cânula única no átrio direito, a temperatura nasofaríngea era baixada até $15^{\circ} \mathrm{C}$ a $18^{\circ} \mathrm{C}$, sendo realizada parada circulatória e removendo-se a cânula venosa. Crianças com mais de $8 \mathrm{~kg}$ foram operadas, ou com parada circulatória, ou com auxílio da circulação extracorpórea, através de canulação da aorta ascendente, das duas veias cavas, através do átrio direito, ou canulação direta destas, com hipotermia moderada $\left(26^{\circ} \mathrm{C}\right.$ a $\left.28^{\circ} \mathrm{C}\right)$ e períodos de hipofluxo.

A correção cirúrgica foi realizada através do átrio direito. Inicialmente, o teste da valva atrioventricular com injeção, sob pressão, de soro no ventrículo esquerdo.

A comunicação interventricular era corrigida inicialmente com retalho de Teflon ou Dacron. Em parte dos pacientes, o mesmo retalho era utilizado para a sutura das cúspides da valva AV e o fechamento da comunicação interatrial (técnica de 1 retalho). Adotou-se, nos demais pacientes, a técnica de dois retalhos; para o fechamento da CIV era utilizado Teflon ou Dacron, sendo as cúspides suturadas ao retalho e a comunicação interatrial (CIA) corrigida com um segundo retalho de Dacron, pericárdio autólogo, ou pericárdio bovino. O seio coronário ficava drenando para o átrio esquerdo, com exceção de casos em que havia veia cava superior esquerda drenando para o seio coronário. Seccionavam-se as cúspides da valva AV, para melhor exposição da CIV quando o cirurgião considerava necessário.

Todas as anomalias cardiovasculares associadas foram corrigidas no mesmo ato cirúrgico. Quan- do havia persistência do canal arterial, era ligado logo antes do início da circulação extracorpórea (CEC). As demais anormalidades cardíacas foram corrigidas durante os períodos de pinçamento aórtico.

Nos quatro primeiros anos da experiência, a técnica de proteção miocárdica foi a de vários períodos da parada isquêmica hipotérmica do coração. A partir de 1978, a cardioplegia hipotérmica (St. Thomas I) vem sendo utilizada em todos os pacientes como método de proteção miocárdica. Esta foi administrada diretamente na aorta ascendente, na dose inicial de $20 \mathrm{ml} / \mathrm{kg}$, sendo repetida metade dessa dose a cada 30 minutos. Usou-se a hipotermia tópica com soro gelado na cavidade pericárdica como medida adicional de proteção miocárdica.

\section{ANÁLISE ESTATÍSTICA}

Os dados de observação deste trabalho foram analisados estatisticamente de acordo com a seguinte metodologia:

A comparação entre os diversos grupos na análise univariada, através do cálculo do "Odds Ratio" (OR) ou Razão de Chance, teve sua significância estatística calculada através do teste do Qui-quadrado. Para algumas variáveis (contínuas) foi realizado, também, o teste $\mathrm{T}$ de Student. Consideravam-se estatisticamente significativos os valores de iguais ou inferiores a 0,05 .

Objetivando analisar o efeito simultâneo de todas as variáveis, realizou-se a análise multivariada de regressão logística 33,125 . O objetivo desta análise é investigar o efeito de cada fator de risco na ocorrência de morte operatória, controlado por eventuais fatores de confusão. As variáveis foram incluídas neste modelo através de um procedimento stepwise, com a tolerância de entrada de alfa $=0,1$, e sua contribuição para a predição de morte foi calculada mediante o teste likelihood ratio, com a significância mínima de 0,05 . O modelo final, controlado pelos dois fatores de confusão, foi sustentado contra todas as variáveis estudadas. Como pacote estatístico, utilizou-se o EGRET.

\section{RESULTADOS}

Dos 52 pacientes operados, $11(21,15 \%)$ faleceram no hospital. A principal causa de óbito foi de origem cardíaca em $9(81,81 \%)$, seguida de mediastinite (1 caso) e neurológica (1 caso). Todos os pacientes que faleceram, assim como as causas dos óbitos, estão listados na Tabela 1. 
SAADI, E. K.; SHORE, D.; LINCOLN, C. - Análise dos fatores de risco na correção cirúrgica do defeito septal atrioventricular de forma total. Rev. Bras. Cir. Cardiovasc., 8(2):136-151, 1993.

TABELA 1

ÓBITOS HOSPITALARES NO DSAVT

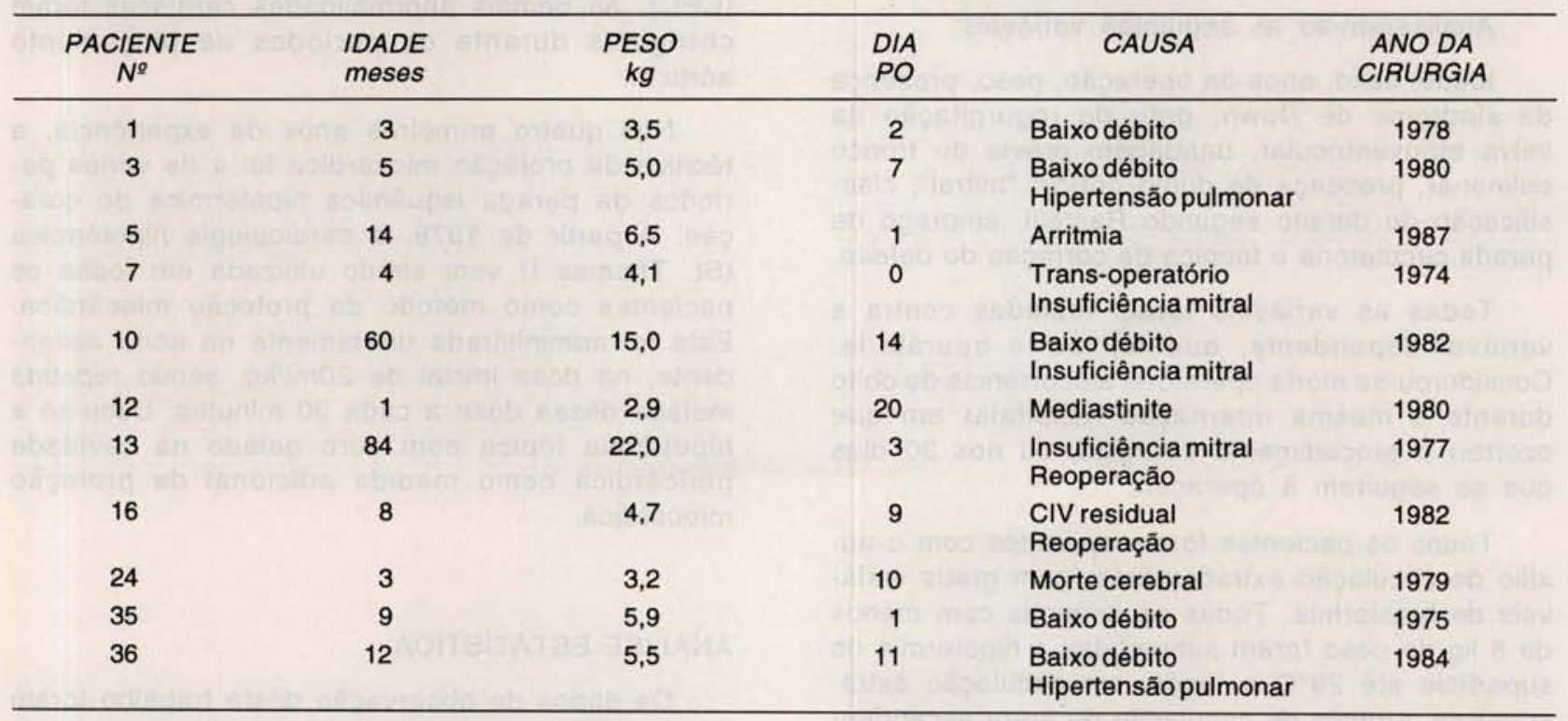

Com relação ao sexo, $35 \%$ dos pacientes do sexo masculino faleceram, enquanto apenas $12,5 \%$ das meninas foram a óbito (NS).

Até 1980 , a mortalidade era de $38,88 \%$; após 1980 , a mortalidade baixou para $11,76 \%$ NS $(p=0,0547)$.

A idade variou de 1 mês a 7 anos, com a média de 24 meses. A idade, na operação, não foi significativa para a ocorrência de morte $(p=0,085)$.

\section{Peso na Cirurgia}

O peso na época da operação variou de $2,9 \mathrm{~kg}$ a $22 \mathrm{~kg}$, para a média de $8,67 \mathrm{~kg}$.

A correlação do peso (em $\mathrm{kg}$ ), no momento da operação, com a mortalidade está apresentada na Tabela 2.

TABELA 2

PESO NA CIRURGIA $(\mathrm{kg})$

\begin{tabular}{|c|c|c|c|}
\hline PESO & $\begin{array}{l}\text { NÜMERO DE } \\
\text { PACIENTES }\end{array}$ & & $\begin{array}{r}\text { TOS } \\
\%\end{array}$ \\
\hline Até $5,0 \mathrm{~kg}$ & 16 & 7 & 43,75 \\
\hline De 5,1 a $10,0 \mathrm{~kg}$ & 16 & 3 & 18,75 \\
\hline Superior a $10,0 \mathrm{~kg}$ & 19 & 1 & 5,26 \\
\hline Total & $51^{*}$ & 11 & \\
\hline
\end{tabular}

$p=0,2177$ NS - Não significativo.

* Em um caso não foi possível a obtençāo do peso.
A mortalidade global de crianças até $5 \mathrm{~kg}$ foi de $43,75 \%$. Entre 5,1 e $10 \mathrm{~kg}$ foi de $18,75 \%$ e acima dos $10 \mathrm{~kg}$ foi de $5,26 \%$. Houve tendência de maior mortalidade nos pacientes de menor peso, porém não ocorreu diferença estatisticamente significativa entre os grupos, para p igual ou inferior a 0,05 , na análise univariada, quando o teste Qui-quadrado foi empregado. No entanto, quando usado o este $\mathrm{T}$ de 
SAADI, E. K.; SHORE, D.; LINCOLN, C. - Análise dos fatores de risco na correção cirúrgica do defeito septal atrioventricular de forma total. Rev. Bras. Cir. Cardiovasc., 8(2):136-151, 1993.

Student, sendo o peso analisado como variável contínua, a diferença foi estatisticamente significativa $(p=0,049)$. Na regressão logística (análise multivariada), o peso se mostrou como fator de risco importante para a ocorrência de morte, funcionando com variável independente (OR $=0,8156$ ), sendo que cada $\mathrm{kg}$ de peso a mais em determinado paciente oferece a proteção de 0,8156 (Tabela 8 ).

A correlação do peso e da técnica de correção com a probabilidade de morte está representada no Gráfico 1.

\section{Insuficiência da Valva Atrioventricular}

A insuficiência severa da valva atrioventricular estava presente, no pré-operatório, em $15(28,85 \%)$ pacientes, sendo que $5(33,33 \%)$ deste grupo faleceram no período per-operatório.

Dos $37(71,15 \%)$ pacientes que não apresentavam insuficiência severa da valva atrioventricular no pré-operatório, $6(16,22 \%)$ faleceram (Tabela 3$)$. Esta diferença não é estatisticamente significativa para $\mathrm{p}=0,05$.

TABELA 3

INSUFICIÊNCIA DA VALVA AV NO PRÉ-OPERATÓRIO

\begin{tabular}{lcrrr}
\hline INSUFICIÊNCIA & \multicolumn{2}{c}{ PACIENTES } & \multicolumn{2}{c}{ ÓBITOS } \\
DA VALVA AV & $N^{Q}$ & \multicolumn{1}{c}{$\%$} & \multicolumn{1}{c}{$N^{Q}$} & \multicolumn{1}{c}{$\%$} \\
\hline Com regurgitação severa & 15 & 28,85 & 5 & 33,33 \\
Sem regurgitação severa & 37 & 71,15 & 6 & 16,22 \\
Total & 52 & 100,00 & 11 & \\
\hline
\end{tabular}

$p=0,3200$ NS - Năo significativo.

\section{Bandagem Prévia do Tronco Pulmonar}

Sete $(13,46 \%)$ pacientes haviam sido submetidos previamente à bandagem do tronco pulmonar, e não houve mortalidade neste grupo durante 0 reparo definitivo.

Dos 45 pacientes restantes, que não haviam sido submetidos a bandagem previamente, 11 $(24,44 \%)$ morreram (Tabela 4$)$. A diferença não é significativa, do ponto de vista estatístico, para $p=0,05$.

\section{Pressão Sistólica do Tronco Pulmonar}

A pressão do tronco pulmonar foi medida, no pré-operatório, em 35 pacientes. Observou-se redução da mortalidade cirúrgica quando a pressão sistólica do tronco pulmonar foi menor do que 40 $\mathrm{mmHg}$ (Tabela 5). Houve diferença estatisticamente
TABELA 4

BANDAGEM PRÉVIA DO TRONCO PULMONAR NO DSAVT

\begin{tabular}{crrrr}
\hline BANDAGEM PRÉVIA & \multicolumn{2}{c}{ PACIENTES } & \multicolumn{2}{c}{ ÓBITOS } \\
& \multicolumn{1}{c}{$N^{0}$} & \multicolumn{1}{c}{$\%$} & \multicolumn{1}{c}{$N^{0}$} & \multicolumn{1}{c}{$\%$} \\
\hline Sim & 7 & 13,46 & 0 & 0,00 \\
Não & 45 & 86,54 & 11 & 24,44 \\
Total & 52 & 100,00 & 11 & \\
\hline
\end{tabular}

$p=0,3292$ NS - Não significativo.

significativa $(p=0,0342)$ na análise univariada. $\mathrm{Na}$ análise multivariada, essa pressão não demonstrou, como variável isolada, aumentar a possibilidade de morte (Tabela 8).

TABELA 5

PRESSÃO SISTÓLICA DO TRONCO PULMONAR

\begin{tabular}{lrrrr}
\hline PRESSÃO SISTÓLICA & \multicolumn{2}{c}{ PACIENTES } & \multicolumn{2}{c}{ ÓBITOS } \\
DO TRONCO PULMONAR & \multicolumn{1}{c}{$N^{9}$} & \multicolumn{1}{c}{$\%$} & \multicolumn{1}{c}{$N^{9}$} & \multicolumn{1}{c}{$\%$} \\
\hline Até $40 \mathrm{mmHg}$ & 12 & 23,08 & 0 & 0,00 \\
De 41 a $70 \mathrm{mmHg}$ & 16 & 30,77 & 6 & 37,50 \\
Superior a $70 \mathrm{mmHg}$ & 7 & 13,46 & 3 & 42,86 \\
Não medida & 17 & 32,69 & 2 & 11,76 \\
Total & 52 & 100,00 & 11 & \\
\hline
\end{tabular}

$p=0,342$ Significativo para $p(0,05)$

\section{Presença da Síndrome de Down}

Dos 52 pacientes, $21(40,38 \%)$ eram portadores de síndrome de Down. Destes, 5 (23,8\%) faleceram.

Dos $31(59,62 \%)$ pacientes que não apresentavam síndrome de Down, a mortalidade foi de 6 $(19,35 \%)$. Não existe diferença estatisticamente significativa para $\mathrm{p}=0,05$.

\section{Presença de Anomalias Associadas}

Onze $(21,15 \%)$ pacientes apresentavam anomalias do sistema cardiovascular associadas ao DSAVT, havendo $2(18,18 \%)$ óbitos. Tais anomalias consistiam de persistência do canal arterial (PCA), comunicação interatrial (CIA) tipo ostium secundum, veia cava superior esquerda, continuação de veia cava inferior via veia ázigos, átrio único, coarctação da aorta, estenose aórtica e obstrução de via de saída de ventrículo direito (VD); em 41 (78,85\%) não foram encontradas anomalias, tendo ocorrido 9 $(21,95 \%)$ óbitos nesta série. Não houve aumento da mortalidade hospitalar na presença de anomalias associadas. 
SAADI, E. K.; SHORE, D.; LINCOLN, C. - Análise dos fatores de risco na correção cirúrgica do defeito septal atrioventricular de forma total. Rev. Bras. Cir. Cardiovasc., 8(2):136-151, 1993.

\section{Classificação do Defeito Segundo Rastelli}

A correlação do tipo de defeito, classificado, segundo Rastelli, em A, B ou C, com a mortalidade hospitalar está apresentada na Tabela 6 . Não houve diferença estatisticamente significativa para $p=0,05$.

TABELA 6

CLASSIFICAÇÃO DO DEFEITO SEGUNDO RASTELLI

\begin{tabular}{lrrrrr}
\hline CLASSIFICAÇÃO & \multicolumn{2}{c}{ PACIENTES } & \multicolumn{2}{c}{ ÓBITOS } \\
DO DEFEITO & \multicolumn{1}{c}{$N^{2}$} & \multicolumn{1}{c}{$\%$} & \multicolumn{1}{c}{$N^{\varrho}$} & \multicolumn{1}{c}{$\%$} \\
\hline Tipo A & 31 & 59,62 & 7 & 22,58 \\
Tipo B & 9 & 17,31 & 0 & 0,00 \\
Tipo C & 12 & 23,07 & 4 & 33,33 \\
Total & 52 & 100,00 & 11 & \\
\hline
\end{tabular}

$p=0,1721$ NS - Não significativo

\section{Presença de Duplo Orifício Mitral}

Em $2(3,85 \%)$ casos desta série, constatou-se, no intra-operatório, duplo orifício mitral. Não houve mortalidade neste grupo. A diferença não foi estatisticamente significativa para $p=0,05$

\section{Técnica de Correção (1 ou 2 Retalhos)}

Adotou-se a técnica cirúrgica com o emprego de um único retalho para a correção da comunicação interventricular e interatrial em $20(38,46 \%)$ pacientes. A mortalidade, neste grupo, foi de 7 pacientes $(35 \%)$.

Em $32(61,54 \%)$ casos utilizaram-se dois retalhos para o fechamento em separado da comunicação interventricular e interatrial. A mortalidade, neste grupo, foi de $4(12,50 \%)$ pacientes. Não houve diferença estatisticamente significativa entre os dois grupos, para $p=0,05$, na análise univariada (Tabela 7). Na análise multivariada (regressão logística), a técnica de correção com um retalho aumentou significativamente, como variável isolada, a mortalidade cirúrgica $(O R=4,909$; Tabela 8).

A correlação da técnica empregada e o peso com a probabilidade de morte está representada no Gráfico 1.

TABELA 7

ANÁLISE UNIVARIADA DOS FATORES DE RISCO PARA MORTE NA CORREÇÃO DO DSAVT

\begin{tabular}{|c|c|c|c|c|}
\hline VARIÁVEL & NÍVEL & TAXA DE MORTALIDADE (\%) & OR & $P$ \\
\hline Sexo & $\begin{array}{l}\text { Masculino } \\
\text { Feminino }\end{array}$ & $\begin{array}{l}35 \\
12,5\end{array}$ & $\begin{array}{l}3,77 \\
1\end{array}$ & NS $(0,1132)$ \\
\hline Ano da operação & $\begin{array}{l}\text { Até } 1980 \\
\text { Após } 1980\end{array}$ & $\begin{array}{l}38,88 \\
11,76\end{array}$ & $\begin{array}{l}4,77 \\
1\end{array}$ & NS $(0,0547)$ \\
\hline Peso & $\begin{array}{l}\text { Até } 5 \mathrm{~kg} \\
5,1 \text { a } 10 \mathrm{~kg} \\
\text { Acima de } 10 \mathrm{~kg}\end{array}$ & $\begin{array}{l}43 \\
18,75 \\
5,26\end{array}$ & $\begin{array}{l}6,22 \\
1,85 \\
1\end{array}$ & $\begin{array}{l}\text { NS }(0,2177) \\
\text { NS }(0,9456)\end{array}$ \\
\hline Síndrome de Down & $\underset{\text { Não }}{\text { Sim }}$ & $\begin{array}{l}23,88 \\
19,35\end{array}$ & 1,30 & NS $(0,9682)$ \\
\hline $\begin{array}{l}\text { Regurgitação severa } \\
\text { da valva do AV }\end{array}$ & $\begin{array}{l}\text { Sim } \\
\text { Não }\end{array}$ & $\begin{array}{l}33,33 \\
16,22\end{array}$ & $\begin{array}{l}2,58 \\
1\end{array}$ & NS $(0,3200)$ \\
\hline $\begin{array}{l}\text { Bandagem do tronco } \\
\text { pulmonar }\end{array}$ & $\begin{array}{l}\text { Sim } \\
\text { Não }\end{array}$ & $\begin{array}{c}0 \\
24,44\end{array}$ & $:$ & NS $(0,3292)$ \\
\hline Anomalias associadas & $\begin{array}{l}\text { Sim } \\
\text { Não }\end{array}$ & $\begin{array}{l}18,18 \\
21,95\end{array}$ & $\begin{array}{l}0,79 \\
1\end{array}$ & NS $(1,000)$ \\
\hline $\begin{array}{l}\text { Pressão sistólica } \\
\text { tronco pulmonar }\end{array}$ & $\begin{array}{l}\text { Até } 40 \mathrm{mmHg} \\
41 \text { a } 70 \mathrm{mmHg} \\
\text { Acima de } 70 \mathrm{mmHg}\end{array}$ & $\begin{array}{c}0 \\
37,50 \\
42,86\end{array}$ & $:$ & 0,0342 \\
\hline $\begin{array}{l}\text { Duplo orifício } \\
\text { "mitral" }\end{array}$ & $\begin{array}{l}\text { Sim } \\
\text { Não }\end{array}$ & $\begin{array}{l}0 \\
22,00\end{array}$ & - & NS $(1,000)$ \\
\hline $\begin{array}{l}\text { Classificação } \\
\text { de Rastelli }\end{array}$ & $\begin{array}{l}\text { A } \\
\text { B } \\
\text { C }\end{array}$ & $\begin{array}{c}22,58 \\
0 \\
33,33\end{array}$ & $:$ & NS $(0,1721)$ \\
\hline Parada circulatória & $\begin{array}{l}\text { Sim } \\
\text { Não }\end{array}$ & $\begin{array}{r}36,00 \\
7,40\end{array}$ & $\begin{array}{l}7,03 \\
1\end{array}$ & 0,0291 \\
\hline Técnica de correção & $\begin{array}{l}1 \text { retalho } \\
2 \text { retalhos }\end{array}$ & $\begin{array}{l}35,00 \\
12,50\end{array}$ & $\begin{array}{l}3,77 \\
1\end{array}$ & NS $(0,1132)$ \\
\hline
\end{tabular}

OR - "Odds Ratio" 
SAADI, E. K.; SHORE, D.; LINCOLN, C. - Análise dos fatores de risco na correção cirúrgica do defeito septal atrioventricular de forma total. Rev. Bras. Cir. Cardiovasc., 8(2):136-151, 1993.

\section{Emprego de Parada Circulatória}

A parada circulatória foi utilizada em 25 (48,08\%) pacientes. Destes, 9(36\%) faleceram. Em 27 $(51,92 \%)$ não se empregou a parada circulatória, e a mortalidade, neste grupo, foi de $2(7,40 \%)$ casos. Esta diferença mostrou ser significativa do ponto de vista estatístico, para $p=0,05$ na análise univariada $(p=0,0291)$.

$\mathrm{Na}$ análise multivariada com regressão logística, livre da ação de outras variáveis, esta variável não se mostrou fator significativo para a morte hospitalar (Tabela 8).

TABELA 8

ANÁLISE MULTIVARIADA (REGRESSĀO LOGÍSTICA) DOS FATORES DE RISCO DE MORTE HOSPITALAR NA CORREÇÄO CIRÚRGICA DO DSAVT

\begin{tabular}{cll}
\hline FATOR DE RISCO & OR & \multicolumn{1}{c}{ LC-95\% } \\
\hline Retalho & 4,909 & $1,079-22,33$ \\
Peso & 0,8156 & $0,6712-0,9911$ \\
\hline
\end{tabular}

OR - "Odds Ratio"

LC - Limite de confiança

Equaçāo de regressão logística

$z=0,5007-0,2038 \times$ peso $+1,591 \times()$ (2 retalho) 1 (1 retalho)

\section{COMENTÁRIOS}

A cirurgia para a correção do DSAVT foi descrita, inicialmente, por MALONEY Jr. et alii 71 e GERBODE et alii ${ }^{40}$, mas foi RASTELLI et alii 98,99 que impuseram um grande impulso em seu tratamento cirúrgico. O conhecimento das características anatômicas deste defeito é um dos pré-requisitos para a correção bem sucedida. Desde o trabalho clássico de LEV ${ }^{65}$, em 1958 , descrevendo a localização do feixe de His no DSAVT, o reparo tem sido feito no sentido de evitar a lesão do tecido de condução. PICCOLLI et alii ${ }^{91}$, em 1978, acrescentaram importantes considerações anatômicas, principalmente com relação às cúspides da valva ${ }^{12} \mathrm{AV}$, aos estudos de Rastelli e Lev. Em 1982, BECKER e ANDERSON ${ }^{12}$ demonstraram a importância prática da descrição morfológica e não embriológica de tais defeitos, denominando-os de defeitos septais atrioventriculares.

As primeiras experiências cirúrgicas para correção deste defeito foram associadas com alta mortalidade cirúrgica (maior que $60 \%$ ), devida, freqüentemente, a lesões do tecido de condução, regurgitação severa da valva AV ou estenose subaórtica 73. Estes resultados foram logo suplantados por relatos na literatura, de mortalidade variando de $0 \%$ a $35 \% 15,28,51,70,76$. Curiosamente, em muitas das primeiras operações realizadas, a técnica de dois retalhos para corrigir o defeito foi utilizada 73 . Os aitores empregavam incisões separadas no átrio direito e ventrículo direito. Em 1962, MALONEY Jr. et alii ${ }^{71}$ descreveram 2 casos em que apenas um retalho foi utilizado para corrigir ambos os defeitos (CIA e CIV) e suspender a valva AV. Mais recentemente, MORENO-CABRAL \& SHUMWAY 80 descreveram a técnica de correção com dois retalhos através da atriotomia direita. CARPENTIER 22 enfatizou a importância da valva $A V$ esquerda ser mantida com sua estrutura de três cúspides, para preservar a sua função, além da utilização da técnica de dois retalhos. Como resultado de todos estes avanços, reduziu-se o risco da correção cirúrgica deste defeito. A necessidade do reparo cirúrgico precoce $e$, mais recentemente, durante o primeiro ano de vida, tem ganho grande aceitação $13,55,58$, 127. Tal abordagem deve-se ao desenvolvimento precoce e acelerado da doença vascular pulmonar, nesta condição, e à melhora nos resultados cirúrgicos $1,14,15,44,58,88,102,127$.

Apesar da mortalidade cirúrgica na correção do DSAVT ter diminuído significativamente na última década, o tratamento cirúrgico permanece um desafio $102,114,127$. O tratamento paliativo, com bandagem do tronco pulmonar, que, no passado, apresentava mortalidade de cerca de $50 \%$, ainda nos dias de hoje tem mortalidade de $5 \%$ a $10 \%$, mesmo em pacientes com insuficiência leve da valva $\mathrm{AV} 37,68$, 103, 108, 129. A mortalidade combinada da cirurgia paliativa com a correção total torna a cirurgia corretiva precoce a melhor alternativa $13,15,18,25,56,57$, $59,68,127$

A mortalidade cirúrgica aumenta sob certas circunstâncias, que devem ser identificadas, porque, mesmo nos dias de hoje, ela ainda não se aproxima de zero na maioria dos centros ${ }^{56}$. A principal causa de morte, na correção cirúrgica das cardiopatias congênitas, é o baixo débito cardíaco 19,56 .

A maioria dos artigos, na literatura, que analisam os fatores de risco para morte hospitalar deixam de distinguir, em suas análises, as formas parciais do DSAV com as formas totais do defeito $24,75,94,114$

A data da operação, em muitas instituições, constitui importante determinante do risco, porque os resultados cirúrgicos vêm melhorando com o tempo 56, 114 . Em nosso estudo, até 1980 , a mortalidade cirurgica era de $38,88 \%$, tendo baixado para $11,76 \%$ após essa data. Tal diferença não é estatisticamente significativa. BERGER et alii ${ }^{15}$ demonstraram que a data da operação, como variável con- 
SAADI, E. K.; SHORE, D.; LINCOLN, C. - Análise dos fatores de risco na correção cirúrgica do defeito septal atrioventricular de forma total. Rev. Bras. Cir. Cardiovasc., 8(2):136-151, 1993.

tínua, estava significativamente relacionada à probabilidade de morte hospitalar em 27 crianças de 2 anos de idade. STUDER et alii 114 identificaram a dada da operação como fator importante de aumento da mortalidade, em 310 pacientes em todas as formas de DSAV.

A baixa idade na cirurgia, assim como o baixo peso, tem sido fator de aumento no risco operatório 15. A mortalidade, para o grupo de pacientes com menos de 2 anos de idade, tem variado de $0 \%$ a $43 \%$; enquanto para crianças menores de 1 ano de idade varia de $3 \%$ a $100 \% 15,28,70,76,127$. Em análise de 27 pacientes com DSAVT com menos de 2 anos de idade, BERGER et alii ${ }^{15}$ não encontraram relação da idade com a mortalidade. STUDER et alii 114 demonstraram que a baixa idade foi importante fator de risco no passado; porém, desde 1976 hoive diminuição deste risco, com o tempo. Em 1987, McGRATH \& GONZALE-LAVIN ${ }^{75}$, em experiência de 54 casos de DSAVT, identificaram a baixa superfície corporal como determinante importante da mortalidade nesta série. Em nosso estudo, a idade não foi fator de risco significativo para morte hospitalar. No entanto, houve maior mortalidade em crianças de baixo peso, especialmente no grupo com menos de $5 \mathrm{~kg}$ peso. O baixo peso na operação demonstrou ser uma variável independente para a ocorrência de morte hospitalar na correção cirúrgica do DSAVT, no presente estudo (análise multivariada).

A insuficiência severa da valva atrioventricular no pré-operatório tem constituído fator de aumento de mortalidade cirúrgica, segundo alguns autores 56, 114; McGRATH \& GONZALES-LAVIN 75 demonstraram, posteriormente, que a severidade da regurgitação AV no pré-operatório não contribuía para o aumento da mortalidade. Alguns autores relatam, inclusive, sua experiência com o uso de próteses mitrais quando é possível a correção com valvoplastia 23,53. Em nosso estudo houve tendência de maior mortalidade no grupo com insuficiência severa da valva AV; porém, do ponto de vista estatístico, a diferença não foi significativa $(p=0,3200)$.

Durante as últimas décadas, cardiologistas, cirurgiōes e patologistas têm tentado determinar qual o melhor momento de intervenção no DSAVT, antes do desenvolvimento da doença vascular pulmonar obstrutiva ${ }^{26}$. Há estudos que sugerem serem as alterações vasculares geralmente reversíveis antes dos 2 anos de idade; porém, mais recentemente, foi demonstrada a possibilidade de lesões fixas antes dos 12 meses 14, 28, 83. Vários autores sugerem que crianças com síndrome de Down podem desenvolver alterações mais precoces e mais rápidas 26,63 . Alguns autores não encontraram relação entre hipertensão ou hiper-resistência pulmonar e a mortalidade cirúrgica 15,101 . McGRATH \& GONZALES-
LAVIN ${ }^{75}$ constataram que níveis elevados de resistência pulmonar estavam relacionados com aumento do risco de morte operatória em 77 pacientes com estes dados disponíveis, quando consideradas todas as formas de defeitos septais atrioventriculares. RIZZOLI et alii ${ }^{101}$ chegaram à mesma conclusão e observaram mortalidade significativamente maior no grupo com síndrome de Down. Em nosso estudo, houve tendência de maior mortalidade quando a pressão sistólica de tronco pulmonar foi superior a $70 \mathrm{mmHg}$; não houve óbito no grupo de pacientes com pressão sistólica inferior a $40 \mathrm{mmHg}$ $(p=0,0342)$. Na análise multivariada, entretanto, a pressão sistólica de tronco pulmonar não se mostrou significativa como variável independente, para a ocorrência de morte. Também não houve diferença na mortalidade nos grupos com ou sem síndrome de Down, neste estudo.

As anomalias da valva "mitral", principalmente o duplo orifício "mitral", têm sido associadas com defeitos septais atrioventriculares $30,46,48,64,126$. Por serem elas infreqüentes, existem poucos trabalhos na literatura enfatizando sua importância no tratamento cirúrgico; a mortalidade, porém, nesta situação, tem sido elevada $25,114,126$. Estudos mais recentes demonstram que o reparo de DSAV associado com duplo orifício "mitral" pode ser feito com baixa mortalidade cirúrgica e excelente resultado tardio 48,64 . Em nosso estudo, 2 pacientes apresentavam duplo orifício "mitral", não houve mortalidade.

A importância do emprego da hipotermia profunda com parada circulatória total na correção de cardiopatias congênitas é reconhecida há décadas 10. KIRKLIN et alii ${ }^{55}$, analisando os fatores de risco na correção de várias cardiopatias em crianças com menos de 3 meses de idade, identificaram a parada circulatória como um dos fatores de redução na mortalidade hospitalar. SANTOS et alii ${ }^{102}$ relataram experiência cirúrgica em 15 pacientes com DSAVT com parada circulatória total sem mortalidade. Mais recentemente, a circulação extracorpórea vem sendo utilizada por alguns autores, mesmo em recémnascidos, com excelentes resultados, deixando a parada circulatória para crianças abaixo de $3,5 \mathrm{~kg}$ de peso ${ }^{127}$. No presente estudo, houve maior mortalidade no grupo em que a parada circulatória foi empregada $(p=0,0291)$. No entanto, a análise multivariada demonstrou que a parada circulatório não funcionou como variável independente para maior mortalidade. $O$ baixo peso é que foi identificado como uma das duas variáveis independentes importantes para a ocorrência de morte, além da técnica de correção com um retalho.

STUDER et alii ${ }^{114}$ identificaram a presença de anomalias cardiovasculares associadas ao DSAV como fator de risco para morte hospitalar. RIZZOLI 
SAADI, E. K.; SHORE, D.; LINCOLN, C. - Análise dos fatores de risco na correção cirúrgica do defeito septal atrioventricular de forma total. Rev. Bras. Cir. Cardiovasc., 8(2):136-151, 1993.

et alii ${ }^{101}$ também relataram maior mortalidade nesta situação, mas apenas nas formas parciais do DSAV, não tendo havido aumento de mortalidade na correção de forma total. Mais recentemente, PACIFICO et alii ${ }^{88}$ publicaram uma série de 29 pacientes com DSAVT e anomalias associadas, que foram operados, entre os quais ocorreu apenas um óbito ( $3 \%)$. Em nossa série, não houve aumento de mortalidade na presença de anomalias associadas.

Vários autores apresentam excelentes resultados cirúrgicos utilizando, para a correção cirúrgica do DSAVT, apenas um único retalho $1,13,21,25$. BOVE et alii 18 relataram redução na mortalidade, de $22 \%$ para $11 \%$, quando a técnica de um retalho foi substituída pela de dois retalhos; concluem que - reparo do DSAVT é facilitado pelo uso de dois retalhos, que cria menos distorção e facilita a obtenção de uma valva suficiente. Outros autores também preconizam o uso de dois retalhos e apresentam excelentes resultados $22,80,88,94,101,127$. No presente estudo, a técnica de correção com apenas um retalho acarretou maior mortalidade, funcionando como variável independente na análise multivariada para a ocorrência de morte $(O R=$ $4,909)$.

\section{CONCLUSÕES}

A análise dos dados do presente estudo, com relação aos fatores de risco independente para morte hospitalar, na correção cirúrgica do defeito septal atrioventricular de forma total nos permite concluir que:

- A técnica de correção com um retalho aumentou significativamente a possibilidade de morte operatória.

- O baixo peso, no momento da cirurgia, contribuiu significativamente para maior mortalidade cirúrgica.

- Os dados sugerem que a técnica de correção com um retalho não deve ser utilizada na correção do DSAVT e que o baixo peso na cirurgia é um fator de risco independente para maior mortalidade cirúrgica.

SAADI, E, K., SHORE, D.; LINCOLN, C. - Risk factors analysis in the surgical repair of complete atrioventricular septal defect. Rev. Bras. Cir. Cardiovasc., 8(2):136-151, 1993.

ABSTRACT: Patients with complete atrioventricular septal defects (CAVSD) frequently present with severe heart failure which cannot be controllable medically and pulmonary hypertension in infancy. Just a small number survives without early surgical treatment. For this reason the definitive repair is indicated to improve the disease's natural history. However many factors are responsible for a still high surgical mortality in this condition. In the present study the surgical experience in the correction of CAVSD is reviewed in order to identify potential statistically important risk factors for operative death. Between January 1974 and December 1990, 52 patients with complete atrioventricular septal defects underwent definitive surgical repair at The Royal Brompton and National Heart and Lung Institute. They were retrospectively studied and the following variables analysed: age, weight, sex, year of the operation, Down's syndrome, atrioventricular valve regurgitation, previous pulmonary artery banding, associated anomalies, systolic pulmonary artery pressure, double "mitral" valve orifice, Rastelli's classification, circulatory arrest, and the surgical technique ( $1 \times 2$ patches). All this variables were studied by the univariate analysis and, to determine which factors were independently responsible for the operative risk, multivariate analysis with logistic regression was applied. Multivariate analysis showed that the low weight at operation an 1 patch technique significantly increased surgical mortality.

DESCRIPTORS: atrioventricular septal defect, surgery, risk factors. 
SAADI, E. K.; SHORE, D.; LINCOLN, C. - Análise dos fatores de risco na correção cirúrgica do defeito septal atrioventricular de forma total. Rev. Bras. Cir. Cardiovasc., 8(2):136-151, 1993.

\section{REFERÊNCIAS BIBLIOGRÁFICAS}

1 ABBRUZZESE, P. A.; LIVERMORE, J.; SUNDERLAND, C. O.; NUNLEY, D. L.; ISSEMBERG, H.; KHONSARI, S.; GARCIA, C. E.; STARR, A. - Mitral repair in complete atrioventricular canal: case of correction in early infancy. J. Thorac. Cardiovasc. Surg., 85: 388-395, 1983.

2 ALFIERI, O. \& PLOKKER, M. - Repair of common atrioventricular canal associated with transposition of the great arteries and left ventricular outflow obstruction. J. Thorac. Cardiovasc. Surg., 84: 872$875,1982$.

3 ALFIERI, O. \& SUBRAMANIAN, S. - Successful repair of complete atrioventricular canal with undivided anterior common leaflet in a 6 month old infant. Ann. Thorac. Surg., 19: 92-96, 1975.

4 ALVIZATOS, P.; ANDERSON, R. H.; MACARTNEY, F. J.; ZUBERBUHLER, J. R.; STARK, J. Atrioventricular septal defect with balanced ventricles and malaligned atrial septum: double outlet right atrium: report of two cases. J. Thorac. Cardiovasc. Surg., 89: 295-297, 1985.

5 ALLWORK, S. P. - Anatomical-embryological correlates in atrioventricular septal defect. Br. Heart. J., 47: 419-429, 1982.

6 ANDERSON, R. H.; ZUBERBUHLER, J. R.; PENKOSKE, P. A.; NECHES, W. H. - Of clefts, comissures and things. J. Thorac. Cardiovasc. Surg., 90: 605-610, 1985.

7 ARCINIEGAS, E. - Pediatric cardial surgery. Chicago, Year Book Medical Publishers, 1985. 466 p.

8 ARCINIEGAS, E.; HAKIMI, M.; FAROOKI, Z. Q.; GREEN, E. W. - Results of total correction of Fallot with complete atrioventricular canal. J. Thorac. Cardiovasc. Surg., 81: 768-773, 1981.

9 ARTMAN, M.; PARRISH, M. D.; BOERTH, R. C.; BOUCEK, R. J.; GRAHAM, T. P. - Short-term hemodynamic effects of hydralazine in infants with complete atrioventricular canal defects. Circulation, 69: 949-954, 1984.

10 BAILEY, L. L.; TAKEUCHI, Y.; WILLIAMS, W. G. TRUSLER, G. A.; MUSTARD, W. T. - Surgical management of congenital cardiovascular anomalies with the use of profound hypothermia and circulatory arrest: analysis of 180 consecutive cases. Thorac. Cardiovasc. Surg., 71: 485-492, 1976.

BARGERON, L. M.; ELLIOT, L. P.; SOTO, B.; BREAM, P. R.; CURRY, G. C. - Axial cineangiography in congenital heart disease. Section I: Concept, technical and anatomic considerations. Circulation, 56: 1075$1083,1977$. septal defect: what is in a name? J. Thorac. Cardiovasc. Surg., 83: 461-469, 1982.
13 BENDER, H. W.; HAMMON, J. W.; HUBBARD, S. G.; MUIRHEAD, J.; GRAHAN, T. P. - Repair of atrioventricular malformation in the first year of life. J. Thorac. Cardiovasc. Surg., 84: 515-522, 1982.

14 BERGER, T. J.; BLACKSTONE, E. H.; KIRKLIN, J. W.; BARGERON, L. M.; HAZELRIG, J. B.; TURNER, M. E. - Survival and probability of cure without and with operation in complete atrioventricular canal. Ann. Thorac. Surg., 27: 104-111, 1979.

15 BERGER, T. J.; KIRKLIN, J. W.; BLACKSTONE, E. H. PACIFICO, A. D.; KOUCHOUKOS, N. T. - Primary repair of complete atrioventricular canal in patients less than 2 years old. Am. J. Cardiol., 41: 906-913, 1978.

16 BHARATI, S. \& LEV, M. - The spectrum of common atrioventricular oriffice (canal). Am. Heart J., 86: 553-561, 1973.

17 BLOOM, K. R.; FREEDOM, R. M.; WILLIAMS, C. M.; TRUSLER, G. A.; ROWE, R. D. - Echocardiographic recognition of atrioventricular valve stenosis associated with endocardial cushion defect: pathologic and surgical correlates. Am. J. Cardiol., 44: 1326$1331,1979$.

18 BOVE, E. L.; SONDHEIMER, H. M.; KAVEY, R. E. W.; BYRUM, C. J.; BLACKMAN, M. S. - Results with two-patch technique for repair of complete atrioventricular septal defect. Ann. Thorac. Surg., 38: $157-161,1984$

19 BULL, C.; COOPER, J.; STARK, J. - Cardioplegic protection of the child's heart. J. Thorac. Cardiovasc. Surg., 88: 287-293, 1984.

20 BULL, C.; RIGBY, M. L.; SHINEBOURNE, E. A. - Should management of complete atrioventricular canal defect be influenced by coexistent Down syndrome? Lancet, 1: 1147-1149, 1985.

21 CAPOUYA, E. R.; LACKS, H.; DRINKWATER, D. C.; PEARL, J. M.; MILGALTER, E. - Management of the left atrioventricular valve in the repair of complete atrioventricular septal defects. J. Thorac. Cardiovasc. Surg., 104: 196-203, 1992.

CARPENTIER, A. - Surgical anatomy and management of the mitral component of atrioventricular canal defects. In: ANDERSON, R. H.; SHINEBOURNE, E. A. Paediatric cardiology. London, Churchill Livingstone, 1978. p. 477-490.

23 CASTANEDA, A. R.; NICOLOFF, D. M.; MOLLER, J. H.; LUCAS, R. V. - Surgical correction of complete atrioventricular canal utilizing ball-valve replacement of the mitral valve: technical considerations and results. J. Thorac. Cardiovasc. Surg., 62: 926-931, 1971.

24 CHIH, P. \& CHUN, C. - Surgical treatment of atrioventricular canal malformations. Ann. Thorac. Surg., 43: 150-154, 1987. 
SAADI, E. K.; SHORE, D.; LINCOLN, C. - Análise dos fatores de risco na correçāo cirúrgica do defeito septal atrioventricular de forma total. Rev. Bras. Cir. Cardiovasc., 8(2):136-151, 1993.

CHIN, A. J.; KEANE, J. F.; NORWOOD, W. I.; CASTANEDA, A. R. - Repair of complete common atrioventricular canal in infancy. J. Thorac. Cardiovasc. Surg., 84: 437-445, 1982.

26 CLAPP, S. K.; PERRY, B. L.; FARROOKI, Z. O.; JACKSON, W. L.; KARPAWICH, P. P.; HAKIMI, M.; ARCINIEGAS, E.; GREEN, E. W.; PINSKY, W. W. - Down's syndrome, complete atrioventricular canal, and pulmonary vascular obstructive disease. $J$. Thorac. Cardiovasc. Surg., 100: 115-121, 1990.

27 CLAPP, S. K.; PERRY, B. L.; FAROOKI, Z. Q.; JACKSON, W. L.; KARPAWICH, P. P.; HAKIMI, M.; ARCINIEGAS, E.; GREEN, E. W. - Surgical and medical results of complete atrioventricular canal: a ten year review. Am. J. Cardiol., 59: 454-454, 1987.

28 CULPEPPER, W.; KOLFF, J.; LIN, C. Y.; VITULLO, D.; LAMBERTI, J.; ARCILLA, R. A.; REPLOGLE, R. Complete common atrioventricular canal in infancy: surgical repair and postoperative hemodynamics. Circulation, 58: 550-558, 1978.

29 DANIELSON, G. K.; McMULLAN, M. H.; KINSLEY, R. H.; DUSHANE, J. W. - Successful repair of complete atrioventricular canal associated with dextroversion, common atrium, and total anomalous systemic venous return. J. Thorac. Cardiovasc. Surg., 66: 817-822, 1973.

30 DAVID, I.; CASTANEDA, A. R.; VAN PRAAGH, R. Potentially parachute mitral valve in common atrioventricular canal: pathological anatomy and surgical importance. J. Thorac. Cardiovasc. Surg., 84: 178186, 1982.

31 DEANFIELD, J. E.; LEANAGE, R.; STROOBANT, J.; CHISPIN, A. R.; TAYLOR, J. F. N.; MACARTNEY, F. J. - Use of high kilovoltage filtered beam radiographs for detection of bronchial situs in infants and young children. Br. Heart J., 44: 577-583, 1980.

32 DISKINSON, D. F.; ARNOLD, R.; WILKINSON, J. L. Congenital heart disease among 160480 liveborn children in Liverpool 1960 to 1969: implications for surgical treatment. Br. Heart J., 46: 55-62, 1981.

33 DRAPER, N. R. \& SMITH, H. - Applied regression analysis. 2. ed. New York, John Wiley and Sons, 1981.

34 EBELS, T.; HO, S. Y.; ANDERSON, R. H.; MEIJBOOM, E. J.; EIJGELAAR, A. - The surgical anatomy of the left ventricular outflow tract in atrioventricular septal defect. Ann. Thorac. Surg., 41: 483-488, 1986

35 EBELS, T.; MEIJBOOM, E. J.; ANDERSON, R. H.; LEEUWEN, J. M. S.; LENSTRA, D.; EIJGELAR, A.; BOSSINA K. K.; HOMAN, J. N.; HEIDE, H. - Anatomic and functional obstruction of the outflow tract in atrioventricular septal defects with separate valve orifices (ostium primum septal defects): an echocardiographic study. Am. J. Cardiol., 54: 843-847, 1984.
EMANUEL, R.; SOMERVILLE, J.; INNS, A.; WITHERS, R. - Evidence of congenital heart disease in offsprings of parents with atrioventricular defects. Br. Heart J., 30: 645-651, 1983.

EPSTEIN, M. L.; MOLLER, J. H.; AMPLATZ, K.; NICOLOFF, D. M. - Pulmonary artery banding in infants with complete atrioventricular canal. J. Thorac. Cardiovasc. Surg., 78: 28-31, 1979.

38 FELDT, R. H.; DUSHANE, J. W.; TITUS, J. L. - The atrioventricular conduction system in persistent common atrioventricular canal defect: correlations with electrocardiogram. Circulation, 42: 437-444, 1970.

39 FRATER, R. W. M. - Persistent common atrioventricular cannal: anatomy and function in relation to surgical repair. Circulation, 32: 120-129, 1965.

40 GERBODE, F.; SANCHEZ, P. A.; ARGUERO, R.; KERTH, W. J.; HILL, J. D.; DEVRIES, P. A.; SELZER, A.; ROBINSON, S. J. - Endocardial cushion deffects. Ann. Surg., 166: 486-492, 1967.

41 GOMES, A. S.; NATH, P. H.; SINGH, A.; LUCAS, R. V.; AMPLATZ, K.; NICOLOFF, D. M.; EDWARDS, J. E. - Accessory flaplike tissue causing ventricular outflow obstruction. J. Thorac. Cardiovasc. Surg., 80:211216, 1980 .

42 GOW, R. M.; FREEDOM, R. M.; WILLIAMS, W. G. TRUSLER, G. A.; ROWE, R. D. - Coarctation of the aorta or subaortic stenosis with atrioventricular septal defect. Am. J. Cardiol., 53: 1421-1428, 1984.

43 GRIFFITHS, S. P.; ELLIS, K.; BURRIS, J. O.; BUMENTHAL, S.; BOWMAN F. O.; MALM, J. R. - Postoperative evaluation of mitral valve function in ostium primum defect with cleft mitral valve (partial form of atrioventricular canal). Circulation, 40: 21-29, 1969.

44 HAWORTH, S. G. - Pulmonary vascular bed in children with atrioventricular septal defect: relation between structural and hemodynamic abnormalities. Am. J. Cardiol., 57: 833-839, 1986

45 HEATH, D. \& EDWARDS, J. E. - The pathology of hypertensive pulmonary vascular disease: a description of six grades of structural changes in the pulmonary arteries with special reference to congenital cardiac septal defects. Circulation, 18: $533-547,1958$

46 HO, S. Y.; MILO, S.; ANDERSON, R. H.; MACARTNEY, F. J.; GOODWIN, A.; BECKER, A. E.; WENINK, A. C. G.; GERLIS, L. M.; WILKINSON, J. I. - Straddling atrioventricular valve with absent atrioventricular connection: report of ten cases. Br. Heart J., 47: 344-352, 1982.

47 HO, S. Y.; ROSSI, M. B.; METHA, A. V.; HEGERTY, A.; LENNOS, S.; ANDERSON, R. H. - Heart block and atrioventricular septal defect. Thorac. Cardiovasc. Surg., 33: 362-365, 1985. 
SAADI, E. K.; SHORE, D.; LINCOLN, C. - Análise dos fatores de risco na correção cirúrgica do defeito septal atrioventricular de forma total. Rev. Bras. Cir. Cardiovasc., 8(2):136-151, 1993.

ILBAWI, M. N.; IDRISS, F. S.; DeLEON, S. Y.; RIGGS T. W.; MUSTER, A. J.; BERRY, T. E.; PAUL, M. H. - Unusual mitral valve abnormalities complicating surgical repair of endocardial cushion defects. $J$. Thorac. Cardiovasc. Surg., 85: 697-704, 1983.

JACOBSTEIN, M. D.; FLETCHER, B. D.; GOLDSTEIN, S.; RIEMENSCHNEIDER, T. A. - Evaluation of atrioventricular septal defects by Magnetic Resonance Imaging. Am. J. Cardiol., 55: 1158-1161, 1985.

JARMAKANI, J. M.; GEORGE, B.; WHELLER, J. Ventricular volume characteritics in infants and children with endocardial cushing defects. Circulation, 58: 153-157, 1978.

51 KAHN, D. R.; LEVY, J.; FRANCE, N. E.; CHUNG, K. J.; DACUNHOS, G. C. - Recent results after repair of atrioventricular canal. Thorac. cardiovasc. Surg., 73: 413-415, 1977.

KATZ, N. M.; BLACKSTONE, E. H.; KIRKLIN, J. W.; BRADLEY, E. L.; LEMMONS, J. E. - Suture techniques for atrioventricular valves: experimental study. J. Thorac. Cardiovasc. Surg., 81: 528-536, 1981.

53 KAWASHIMA, Y.; MATSURA, H.; HIROSE, H.; NAKAMO, S.; SHIMAZAKI, Y.; MIYAMOTO, K. Surgical treatment of atrioventricular canal defect with an endocardial cushion prosthesis. Circulation, 68: 139-143, 1983.

KAWASHIMA, Y.; NAKANO, S.; KATO, M.; DANNO, M.; SATO, K.; MANABE, H. - Fate of pericardium utilized for the closure of ventricular septal defect: postoperative ventricular septal aneurysm. J. Thorac. Cardiovasc. Surg., 68: 209-218, 1974.

KIRKLIN, J. K.; BLACKSTONE, E. H.; KIRKLIN, J. W.; MCKAY, R.; PACIFICO, A. D.; BARGERON, L. M. Intracardiac surgery in infants under age 3 months: incremental risk factors for hospital mortality. $A m$. J. Cardiol., 48: 500-506, 1981.

KIRKLIN, J. W.; BARRAT-BOYES, B. G. - Cardiac surgery. New York, John Wiley and Sons, 1986. $1550 \mathrm{p}$.

57 KIRKLIN, J. W. \& BLACKSTONE, E. H. - Management of the infant with complete atrioventricular canal. J. Thorac. Cardiovasc. Surg., 78: 32-34, 1979.

KIRKLIN, J. W.; BLACKSTONE, E. H.; BARGERON, L. M.; PACIFICO, A. D.; KIRKLIN, J. K. - The repair of atrioventricular septal defects in infancy. Int. J. Cardiol., 13: 333-360, 1986.

KIRKLIN, J. W.; BLACKSTONE, E. H.; PACIFICO, A. D.; BROWN, R. N.; BARGERON, L. M. - Routine primary repair vs two-stage repair of tetralogy of Fallot. Circulation, 60: 373-385, 1979.

60. KRONGRAD, E.; MALM, J. R.; BOWMAN, F. O.; HOFFMAN, B. F.; KAISER, G. A.; WALDO, A. L. -
Electrophysiological delineation of the specialized atrioventricular conduction system in patients with congenital heart disease. 1: Delineation of His bundle proximal to the membranous septum. J. Thorac. Cardiovasc. Surg., 67: 875-882, 1974.

61 KRONGRAD, E.; MALM, J. R.; BOWMAN, F. O.; HOFFMAN, B. F.; WALDO, B. A. - Electrophysiological delineation of the specialized A-V conduction system in patients with congenital heart disease. II: Delineation of the distal His bundle and the right bundle branch. Circulation, 49: 1232-1238, 1974

62 LACORTE, M. A.; FELLOWS, K. E.; WILLIAMS, R. G. - Overiding tricuspid valve: echocardiographic and angiocardiographic features. Am. J. Cardiol., 37: 911-919, 1976

63 LAURSEN, H. B. - Congenital heart disease in Down's syndrome. Br. Heart J., 38: 32-35, 1976.

64 LEE, C. N.; DANIELSON, G. K.; SCHAFF, H. V.; PUGA F. J.; MAIR, D. D. - Surgical treatment of double orifice mitral valve in atrioventricular canal defects: experience in 25 patients. J. Thorac. Cardiovasc. Surg., 90: 700-705, 1985.

65 LEV, M. - The architecture of the conduction system in congenital heart disease. I: Common atrioventricular orifice. AMA Arch. Patol., 65: 174-181, 1958.

66 LILLEHAY, C. W.; COHEN, M.; WARDEN, H. E. VARCO, R. L. - The direct-vision intracardiac correction of congenital anomalies by controlled cross circulation: results in thirty-two patients with ventricular septal defects, tetralogy of Fallot, and atrioventricularis communis defects. Surgery, $\mathbf{3 8}$ : 11-19, 1955.

67 MACARTNEY, F. J.; REES, P. G.; DALY, K.; PICCOLI, G. P.; TAYLOR, J. F. N.; DE LEVAL, M. R.; STARK, J.; ANDERSON, R. H. - Angiocardiographic appearances of atrioventricular defects with particular reference to distinction of ostium primum atrial septal defect from common atrioventricular orifice. $\mathrm{Br}$ Heart J., 42: 640-656, 1979.

68 MACARTNEY, F. J.; TAYLOR, J. F. N.; GRAHAM, G. R.; DE LEVAL, M. R.; STARK, J. - The fate of survivors of cardiac surgery in infancy. Circulation, 62: $80-91,1980$

69 MACHADO, M. V. L.; CRAWFORD, D. C.; ANDERSON, R. H.; ALLAN, L. D. - Atrioventricular septal defect in prenatal life. Br. Heart J., 59: 352-355, 1988.

70 MAIR, D. D. \& McGOON, D. C. - Surgical correction of atrioventricular canal during the first year of life. Am. J. Cardiol., 40: 66-69, 1977.

71 MALONEY Jr., J. V.; MARABLE, S. A.; MULDER, D. G. - The surgical treatment of common atrioventricular canal. J. Thorac. Cardiovasc. Surg., 43: 84-90, 1962. 

P. A. - Surgical management of complete atrioventricular canal. J. Thorac. Cardiovasc. Surg., 83: 670-679, 1982.

73 McGOON, D. C.; DUSHANE, J. W.; KIRKLIN, J. W. Surgical treatment of endocardial cushion defects. Surgery, 46: 185-190, 1956.

74 McGOON, D. C.; McMULLAN, M. H.; MAIR, D. D.; DANIELSON, G. K. - Correction of complete atrioventricular canal in infants. Mayo Clin. Proc., 48: $769-772,1973$

75 McGRATH, L. B. \& GONZALEZ-LAVIN, L. - Actuarial survival, freedom from reoperation and other events after repair of atrioventricular septal defects. J. Thorac. Cardiovasc. Surg., 94: 582-590, 1987.

76 MIDGLEY, F. M.; GALIOTO, F. M.; SHAPIRO, S. R.; PERRY, L. W.; SCOTT, L. P. - Experience with repair of complete atrioventricular canal. Ann. Thorac. Surg., 30: 151-159, 1980.

77 MILLIS, N. L.; OSHNER, J. L.; KING, T. D. - Correction of type C complete atrioventricular canal: surgical considerations. J. Thorac. cardiovasc. Surg., 71: 20-28, 1976.

78 MILO, S.; HO, S. Y.; WILKINSON, J. I.; ANDERSON, R. H. - Surgical anatomy and atrioventricular conduction tissues of hearts with isolated ventricular septal defects. J. Thorac. Cardiovasc. Surg., 79: 244-255, 1980.

79 MITCHELL, S. C.; KORONES, S. B.; BERENDES, H. W. - Congenital heart disease in 56.109 births: incidence and natural history. Circulation, 43: 323$332,1971$.

80 MORENO-CABRAL, R. \& SHUMWAY, N. E. - Doublepatch technique for correcting of complete atrioventricular canal. Ann. Thorac. Surg., 33: 88-91, 1982.

81 NATH, P. H.; SOTO, B.; BINI, R. M.; BARGERON, L. M.; PACIFICO, A. D. - Tetralogy of Fallot with atrioventricular canal: an angiographic study. J. Thorac. Cardiovasc. Surg., 87: 421-430, 1984.

82 NEUFELD, H. N.; TITUS, J. L.; DUSHANE, J. W.; BURCHELL, H. B.; EDWARDS, J. E. - Isolated ventricular septal defect of the persistent common atrioventricular canal type. Circulation, 23: 685696, 1961.

83 NEWFELD, E. A.; SHER, M.; PAUL, M. H.; NIKAIDON, $\mathrm{H}$. - Pulmonary vascular disease in complete atrioventricular canal defect. Am. J. Cardiol., 39: 721-726, 1977.

84 NORA, J. J. \& NORA, A. H. - The evolution of specific genetic and environmental counseling in congenital heart disease. Circulation, 57: 205-213, 1978.
85 PACIFICO, A. D. - Surgical treatment of complex atrioventricular septal defects. Cardiol. Clin., 7: 399410, 1989.

86 PACIFICO, A. D. \& KIRKLIN, J. W. - Surgical repair of complete atrioventricular canal with anterior common leaflet attached to an anomalous right ventricular papillary muscle. J. Thorac. Cardiovasc. Surg., 65: 727-730, 1973.

87 PACIFICO, A. D.; KIRKLIN, J. W.; BARGERON, L. M. - Repair of complete atrioventricular canal associated with terralogy of Fallot or double outlet right ventricle: report of 10 patients. Ann. Thorac. Surg., 29: 351$351,1980$.

88 PACIFICO, A. D.; RICCHI, A.; BARGERON, L. M.; COLVIN, E. C.; KIRKLIN, J. W.; KIRKLIN, J. K. Corrective repair of complete atrioventricular canal defects and major associated cardiac anomalies. Ann. Thorac. Surg., 46: 645-651, 1988.

89 PENKOSKE, P. A.; NECHES, W. H.; ANDERSON, R. H.; ZUBERBUHLER, J. R. - Further observations on the morphology of atrioventricular septal defects. J. Thorac. Cardiovasc. Surg., 90: 611-622, 1985.

90 PICOLLI, G. P.; HO, S. Y.; WILKINSON, J. L.; MACARTNEY, F. J.; GERLIS, L. M.; ANDERSON, R. H. - Left-sided obstructive lesions in atrioventricular septal defects: an anatomic study. J. Thorac. Cardiovasc. Surg., 83: 453-460, 1982.

91 PICCOLLI, G. P.; MACARTNEY, F. J.; WILKINSON, J. L.; GERLIS, L. M.; ANDERSON, R. H. - Morphology and classification of complete atrioventricular defects. Br. Heart. J., 42: 633-633, 1978.

PIERONI, D. R.; HOMCY, E.; FREEDOM, R. M. Echocardiography in atrioventricular canal defect: a clinical spectrum. Am. J. Cardiol., 35: 54-58, 1975.

93 PILLAI, R.; HO, S. Y.; ANDERSON, R. H.; LINCOLN, C. - Ostium primum atrioventricular septal defect: an anatomical and surgical review. Ann. Thorac. Surg., 41: $458-461,1986$.

94 POZZI, M.; M.; REMIG, J.; FIMMERS, R.; URBAN, A. E. - Atrioventricular septal defects: analysis of short and medium term results. J. Thorac. Cardiovasc. Surg., 101: 138-142, 1991.

QUAEGEBEUR, J.; KIRKLIN, J. W.; PACIFICO, A. D.; BARGERON, L. M. - Surgical experience with unroofed coronary sinus. Ann. Thorac. Surg., 27: 418$425,1979$.

96 RASTELLI, G. C.; KIRKLIN, J. W.; TITUS, J L. - Anatomic observations on complete form of persistent common atrioventricular canal with special reference to atrioventricular valves. Mayo Clin. Proc., 41: 296$301,1966$.

97 RASTELLI, G. C.; ONGLEY, P. A.; KIRKLIN, J. W.; McGOON, D. C. - Surgical repair of complete form 
SAADI, E. K.; SHORE, D.; LINCOLN, C. - Análise dos fatores de risco na correção cirúrgica do defeito septal atrioventricular de forma total. Rev. Bras. Cir. Cardiovasc., 8(2):136-151, 1993.

of persistent common atrioventricular canal. J. Thorac. Cardiovasc. Surg., 55: 299-304, 1968.

RASTELLI, G. C.; ONGLEY, P. A.; McGOON, D. C. Surgical repair of complete atrioventricular canal with anterior common leaflet undivided and unattached to ventricular septum. Mayo Clin. Proc., 44: 335-341, 1969.

99 RASTELLI, G. C.; ONGLEY, P. A.; TITUS, J. L. - Ventricular septal detect of atrioventricular canal type with straddling right atrioventricular valve and mitral valve deformity. Circulation, 37: 816-825, 1968.

100 RASTELLI, G. C.; WEIDMAN, W. H.; KIRKLIN, J. W. Surgical repair of the partial form of persistent common atrioventricular canal, with special reference to the problem of mitral valve incompetence. Circulation, 31: 31-35, 1965.

101 RIZZOLI, G.; MAZZUCCO, A.; BRUMANA, T.; VALFRE, C.; RUBINO, M.; ROCCO, F.; DALIENTO, L.; FRESCURA, C.; GALLUCCI, V. - Operative risk of correction of atrioventricular septal detects. Br. Heart J., 52: 258-265, 1984.

102 SANTOS, A.; BOUCEK, M.; RUTTENBERG, H.; VEASY, G.; ORSMOND, G.; McGOUGH, E. - Repair of atrioventricular septal defects in infancy. J. Thorac. cardiovasc. Surg., 91: 505-510, 1986.

103 SILVERMAN, N.; LEVITSKY, S.; FISCHER, E.; DUBROW, I.; HASTREITER, A.; SCAGLIOTTI, D. Efficacy of pulmonary artery banding in infants with complete atrioventricular canal. Circulation, 68: 148$153,1983$.

104 SMALLHORN, J. F.; SUTHERLAND, G. R.; ANDERSON, R. H.; MACARTNEY, F. J. - Cross-sectional echocardiographic assessment of conditions with atrioventricular valve leaflets attached to the atrial septum at the same level. Br. Heart J., 48: 331-41, 1982.

105 SMALLHORN, J. F.; TOMMASINI, G.; ANDERSON, R. H.; MACARTNEY, F. J. - Assessment of atrioventricular septal defects by two dimensional echocardiography. Br. Heart J., 47: 109-121, 1982.

106 SMALLHORN, J. F.; TOMMASINI, G.; MACARTNEY, F. J. - Two-dimensional echocardiographic assessment of common atrioventricular valves in univentricular hearts. Br. Heart. J., 46: 30-34, 1981.

107 SMALLHORN, J. F.; DE LEVAL, M. R.; STARK, J.; SOMERVILLE, J.; TAYLOR, J. F. N.; ANDERSON, R. H.; MACARTNEY, F. J. - Isolated anterior mitral cleft: two dimensional echocardiographic assessment and differentiation from clefts associated with atrioventricular septal defect. Br. Heart. J., 48: 109116, 1982.

108 SOMERVILLE, J.; AGNEW, T.; STARK, J.; WATERSTON, D. J.; ABERDEEN, E.; CARTER, B.; WAICH, S. - Banding of the pulmonary artery for common atrioventricular canal. Br. Heart J., 29: 816-828, 1967.
109 SOTO, B.; BARGERON, L. M.; PACIFICO, A. D.; VANINI, V.; KIRKLIN, J. W. - Angiography of atrioventricular canal defects. Am. J. Cardio., 48: 492$499,1981$.

110 SOTO, B.; BECKER, A.; MOULAERT, A. J.; LIE, T. J.; ANDERSON, R. H. - Classification of ventricular septal defects. Br. Heart. J., 43: 332-343, 1980.

111 SRIDAROMONT, S.; FELDT, R.; RITTER, D. G.; DAVIS, G. D.; McGOON, D. C.; EDWARDS, J. E. - Doubleoutlet right ventricle associated with persistent common atrioventricular canal. Circulation, 52: 933942, 1975.

112 STARK, J. - Do we really correct congenital heart defects? J. Thorac. Cardiovasc. Surg., 97: 1-9, 1989.

113 STEWART, S.; HARRIS, P.; MANNING, J. - Complete endocardial cushion defect: operation technique and results. J. Thorac. Cardiovasc. Surg., 78: 914-919, 1979.

114 STUDER, M.; BLACKSTONE, E. H.; KIRKLIN, J. W.; PACIFICO, A. D.; SOTO, B.; CHUNG, G. K. T.; KIRKLIN, J. K.; BARGERON, L. M. - Determinants of early and late results of repair of atrioventricular septal (canal) defects. J. Thorac. Cardiovasc. Surg., 84: 523-542, 1982.

115 SUTHERLAND, G. R.; GODMAN, M. J.; SMALLHORN, J. F.; GUITERRAS, P.; ANDERSON, R. H.; HUNTER, S. - Ventricular septal defects: two dimensional echocardiographic and morphological correlations. Br. Heart J., 47: 316-328, 1982.

116 TENKHOFF, L. \& STAMM, S. J. - An analysis of 35 cases of the complete form persistent common atrioventricular canal. Circulation, 48: 416-427, 1973.

117 THANOPOULOS, B. D.; FISCHER, E. A.; DUBROW, I. W.; HASTREITER, A. R. - Right and left ventricular volumes characteristics in common atrioventricular canal. Circulation, 57: 991-995, 1978.

118 THIENE, G.; WENINK, A. C. C.; FRESCURA, C.; WILKINSON, J. L.; GALLUCI, V.; HO, S. Y.; MAZZUCCO, A.; ANDERSON, R. H. - Surgical anatomy and pathology of the conduction tissues in atrioventricular defects. J. Thorac. Cardiovasc. Surg., 82: 928-937, 1981.

119 UGARTE, M.; SLAMANCA, F. E.; QUERO, M. - Endocardial cushion defects: an anatomical study of 54 specimens. $\mathrm{Br}$. Heart J., 38: 674-682, 1976.

120 URBAN, A. E. - Total correction of complete atrioventricular canal: surgical technique and analysis of long-term results. Prog. Pediatr. Surg., 25: 118$122,1990$.

121 URETZKY, G.; PUGA, F. J.; DANIELSON, G. K.; FELDT, R. H.; JULSRUD, P. R.; SEWARD, J. B.; EDWARDS, 
W. D.; McGOON, D. C. - Complete atrioventricular canal associated with tetralogy of Fallot: morphologic surgical considerations. J. Thorac. Cardiovasc. Surg., 87: 756-766, 1984.

122 VARGAS, F. J.; COTO, E. O.; MAYER, J. E.; JONAS, R. A.; CASTANEDA, A. R. - Complete atrioventricular canal and tetralogy of Fallot: surgical considerations. Ann. Thorac. Surg., 42: 258-265, 1986.

123 VOUHE, P. R. \& NEVEUX, J. Y. - Surgical repair of tetralogy of Fallot with complete atrioventricular canal. Ann. Thorac. Surg., 41: 342-344, 1986.

124 WAKAI, C. S. \& EDWARDS, J. E. - Development and pathologic considerations in persistent common atrioventricular canal. Proc. Mayo Clin., 31: 487-492, 1956.

125 WALKER, S. H. \& DUNCAN, D. B. - Estimation of the probability of an event as a function of several independent variables. Biometrica, 54: 167-179, 1967.

126 WARNES, C. \& SOMERVILLE, J. - Double mitral valve orifice in atrioventricular defects. Br. Heart J., 49: 59-64, 1983.

127 WEINTRAUB, R. G.; BRAWN, W. J.; VENABLES, A. W.; MEE, R. B. B. - Two-patch repair of complete atrioventricular septal defect in the first year of life. J. Thorac. Cardiovasc. Surg., 99: 302-326, 1990.

128 WILLIAMS, R.\& RUDD, M. - Echocardiographic features of endocardial cushing defects. Circulation, 49: 418422, 1974.

129 WILLIAMS, W. H.; GUYTON, R. A.; MICHALIK, R. E.; PLAUTH, W. H.; ZORN-CHELTON, S.; JONES, E. L.; RHEE, K. H.; HATCHER, C. R. - Individualized surgical management of complete atrioventricular canal. J. Thorac. Cardiovasc. Surg., 86: 838-844, 1983.

130 YOKOYAMA, M.; ANDO, M.; TAKAO, A.; SAKAKIBARA, $S$. - The location of the coronary sinus in endocardial cushion defects. Am. Heart J., 85: 302-307, 1973.

131 YOSHIDA, H.; FUNABASHI, T.; NAKAYA, S.; MAEDA, T.; TANIGUCHI, N. - Subxiphoid cross-sectional echocardiographic imaging of the goose neck deformity in endocardial cushion defect. Circulation, 62: 1319-1323, 1980.

132 ZAVANELLA, C.; MATSUDA, H.; SUBRAMANIAN, S. Successful correction of a complete form of atrioventricular canal associated with tetralogy of Fallot: case report. J. Thorac. cardiovasc. Surg., 74: 195-198, 1977. 